Corrigendum

\title{
Corrigendum to "Acute Amiodarone Pulmonary Toxicity after Drug Holiday: A Case Report and Review of the Literature"
}

\author{
Ahmed Abuzaid, ${ }^{1}$ Marwan Saad, ${ }^{2,3}$ Mohamed Ayan, ${ }^{1}$ Amjad Kabach, ${ }^{1}$ \\ Toufik Mahfood Haddad, ${ }^{1}$ Aiman Smer, ${ }^{4}$ and Amy Arouni ${ }^{4}$ \\ ${ }^{1}$ Department of Internal Medicine, Creighton University, Omaha, NE 68131, USA \\ ${ }^{2}$ Department of Internal Medicine, Seton Hall University School of Health and Medical Sciences, Trinitas Regional Medical Center, \\ Elizabeth, NJ 07207, USA \\ ${ }^{3}$ Department of Cardiology, Ain Shams University, Cairo 11381, Egypt \\ ${ }^{4}$ Department of Cardiology, Creighton University, Omaha, NE 68131, USA
}

Correspondence should be addressed to Marwan Saad; s.marwan@aol.com

Received 29 August 2015; Accepted 28 September 2015

Copyright () 2015 Ahmed Abuzaid et al. This is an open access article distributed under the Creative Commons Attribution License, which permits unrestricted use, distribution, and reproduction in any medium, provided the original work is properly cited.

In the paper titled "Acute Amiodarone Pulmonary Toxicity after Drug Holiday: A Case Report and Review of the Literature" [1], the fifth author's name (Toufik Mahfood Haddad) is corrected as follows. First name is Toufik and last name is Mahfood Haddad.

\section{References}

[1] A. Abuzaid, M. Saad, M. Ayan et al., "Acute amiodarone pulmonary toxicity after drug holiday: a case report and review of the literature," Case Reports in Cardiology, vol. 2015, Article ID 927438, 3 pages, 2015. 


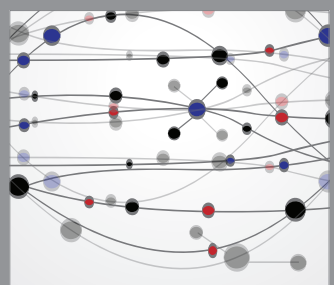

The Scientific World Journal
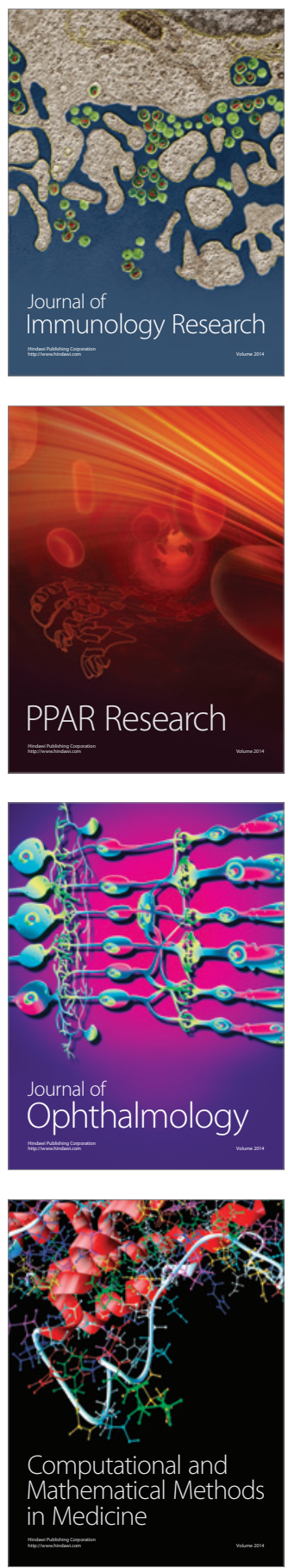

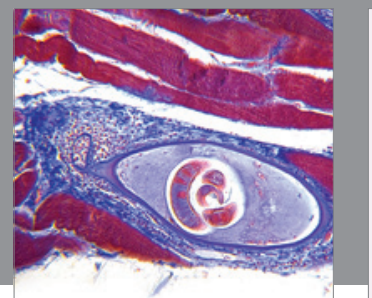

Gastroenterology

Research and Practice
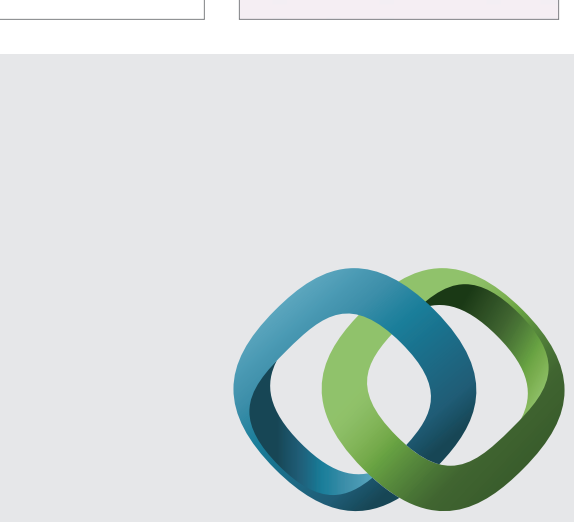

\section{Hindawi}

Submit your manuscripts at

http://www.hindawi.com
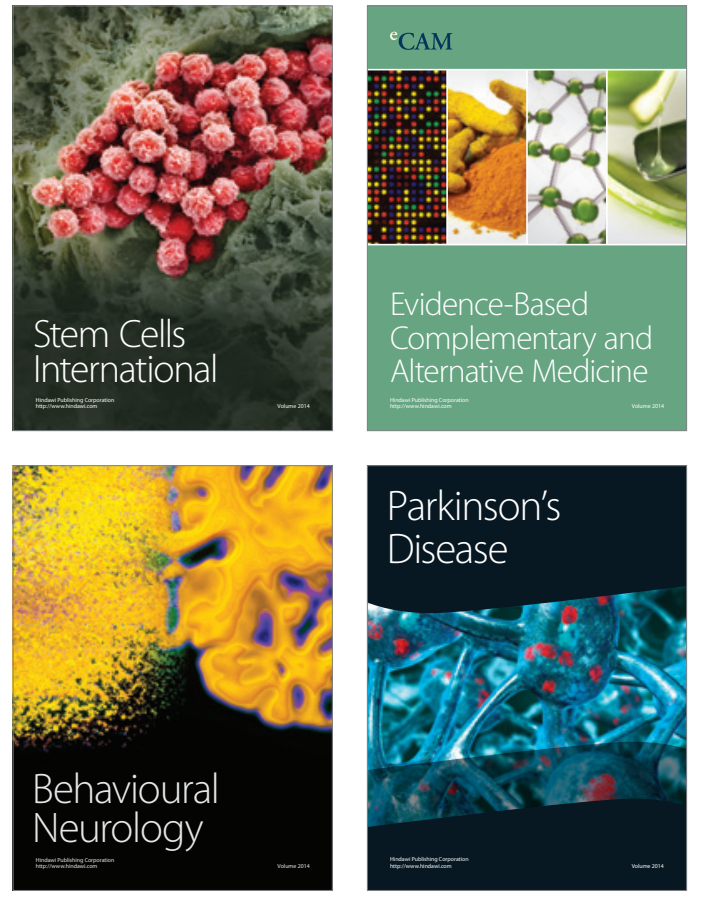
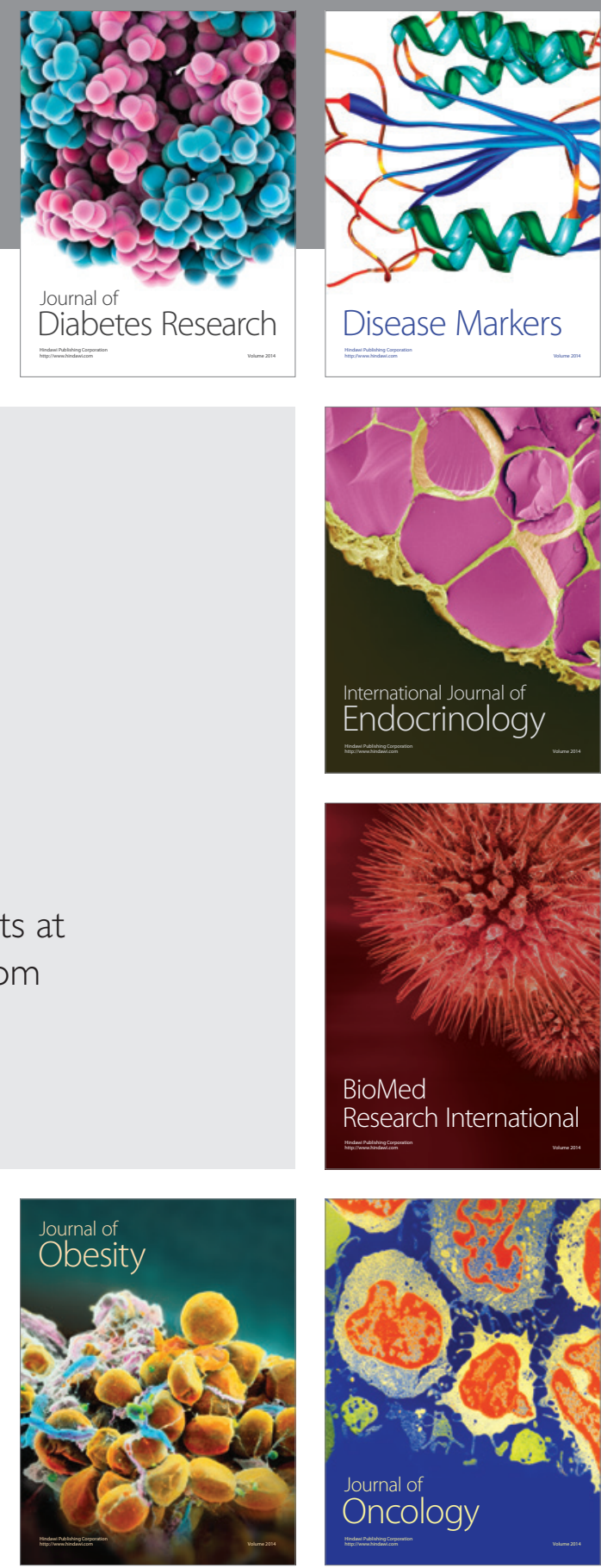

Disease Markers
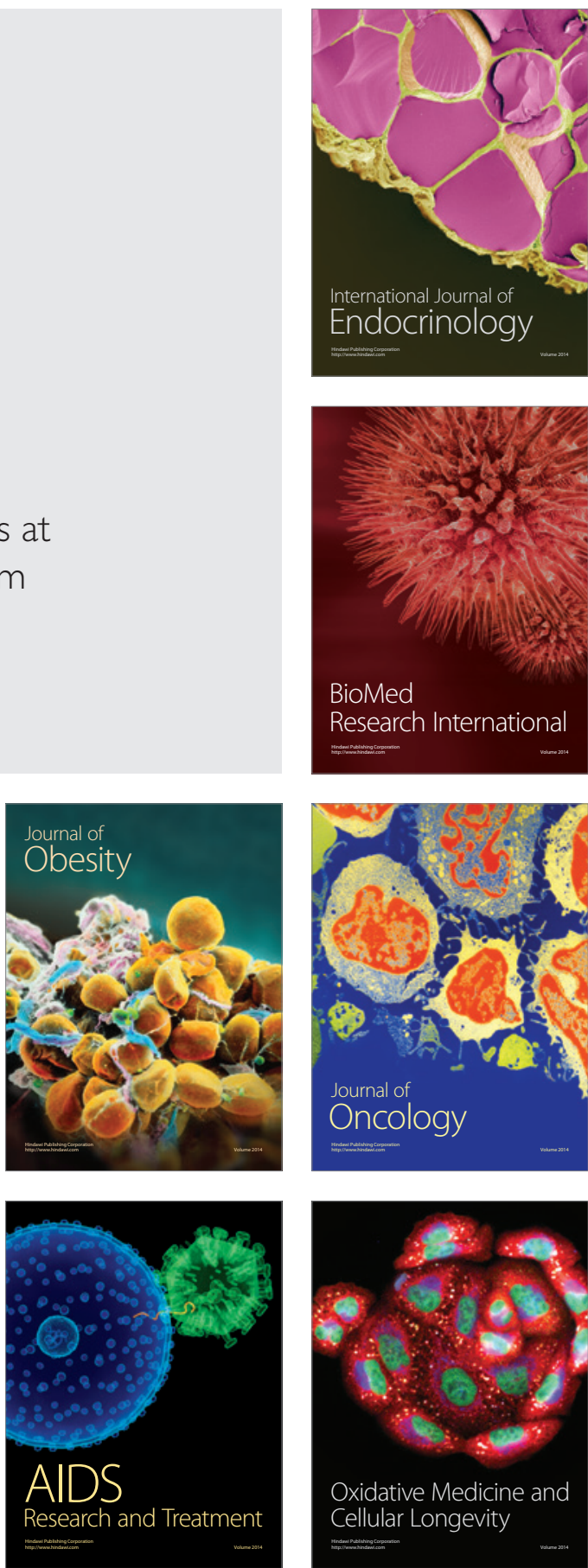